\title{
Cystic fibrosis in Europe: patients live longer but are we ready?
}

\author{
Carsten Schwarz ${ }^{1}$ and Dominik Hartl ${ }^{2}$
}

\begin{abstract}
Affiliations: ${ }^{1}$ Dept of Pediatric Pneumology and Immunology, Cystic Fibrosis Centre Berlin, Charité Universitätsmedizin Berlin, Berlin, Germany. ${ }^{2}$ Dept of Pediatrics I, Comprehensive Cystic Fibrosis Center (CCFC) Tübingen-Stuttgart, University of Tübingen, Tübingen, Germany.
\end{abstract}

Correspondence: Dominik Hartl, Dept of Pediatrics I, Comprehensive Cystic Fibrosis Center (CCFC) HoppeTübingen-Stuttgart, University of Tübingen, Seyler-Strasse 1, Tübingen 72076, Germany.

E-mail: Dominik.hartlamed.uni-tuebingen.de

0 @ERSpublications

This study highlights the future challenge to deal with an ageing CF population and innovative, but expensive drugs http://ow.ly/JRCnx

Having passed the 25th anniversary of discovering the cystic fibrosis transmembrane conductance regulator (CFTR) gene, let's take a look forward to the next 25 years. Newborn screening, animal models, biomarkers, innovative drugs (prototypical CFTR modulators), and advances in lung transplantation have moved the field forward, probably faster than for any other lung disease in recent years [1-5]. As a consequence, the mean survival of patients with cystic fibrosis (CF) is steadily increasing and now exceeds 40 years of age in most European countries [6,7] and beyond [8].

But no progress comes without a price. At least two major changes stir up the CF landscape today. 1) New drugs [9]: a CFTR potentiator (VX-770 known as ivacaftor) entered the market as the first mutation-specific therapy for a subgroup of patients with CF ( $\sim 5 \%$ of patients in Europe, carrying class 3 mutations). However, the costs of this drug pose a challenge for several CF healthcare systems, an issue gaining even broader relevance with the expected introduction of combined CFTR potentiators and correctors for patients with $\Delta \mathrm{F} 508$, the most common mutation in CF [10]. 2) Demographics: the CF population ages with a transition from classical child-disease to adult-disease entity, resulting in a significantly higher proportion of adult CF patients in most industrialised countries [11]. But are we prepared for this change? To assess this issue at the European level, the European Respiratory Society (ERS) joined forces with the European Cystic Fibrosis Society (ECFS) in 2012 and initiated the ERS/ECF Task Force on The Provision of Care for Adults with Cystic Fibrosis in Europe. The findings from this task force are published in the current edition of the European Respiratory Journal [12]. The task force utilised CF patient registries in order to predict the number of CF patients by the year 2025, in 34 European countries. Although relying on population dynamics and statistical models, the findings from this study comprehensively demonstrate that the CF population in Europe will increase significantly (50\%) by the year 2025 . Stratifying estimated patient numbers according to age, the study forecasts that mainly the number of adult CF patients will increase (75\%). The estimated rise in adult CF patients in Europe seems dramatic for some smaller Eastern European countries, which currently have low patient numbers and no established CF infrastructure, e.g. Moldova, Serbia and Slovenia are expected to have increases in CF patients of $212 \%, 168 \%$ and $164 \%$, respectively, by the year 2025 . However, European countries with pre-existing larger CF populations and established CF healthcare systems are predicted to face a substantial increase of their adult CF patients as well, for instance France, Belgium, Spain and Italy. The ERS/ECFS task force went beyond estimating future patient numbers and compared the gross national incomes (GNIs) of these countries. A special light should be shed on countries with a very low GNI. These countries are already facing serious problems in taking care of their CF patient population, e.g. in the

Received: Feb 132015 | Accepted after revision: Feb 162015

Conflict of interest: None declared.

Copyright OERS 2015 
Ukraine where not only adult but also child CF patients are forecasted to increase significantly in the future. These healthcare systems won't be prepared for the demographic change and the financial burden that comes with it.

Methodologically, the findings from this ERS/ECFS task force have highlighted the lack of high-quality patient registries in several European countries, thereby limiting the conclusions drawn from this study and underscoring that registries are indispensable for demographic research and should be promoted throughout Europe.

This first pan-European study of future CF demographics should remind us that early diagnosis and longer survival comes with a cost. It is the imperative responsibility of all CF healthcare professionals across Europe to plan ahead for the CF of tomorrow. To understand the demographics is the first, but should not be the last, step in order to optimise CF care, because patients should not pay the price for growing old with this disease.

\section{References}

Stoltz DA, Meyerholz DK, Welsh MJ. Origins of cystic fibrosis lung disease. N Engl J Med 2015; 372: 351-362.

Pittman JE, Cutting G, Davis SD, et al. Cystic fibrosis: NHLBI workshop on the primary prevention of chronic lung diseases. Ann Am Thorac Soc 2014; 11: Suppl. 3, S161-S168.

3 Mall MA, Hartl D. CFTR: cystic fibrosis and beyond. Eur Respir J 2014; 44: 1042-1054.

4 De Boeck K, Kent L, Davies J, et al. CFTR biomarkers: time for promotion to surrogate end-point. Eur Respir J 2013; 41: 203-216.

5 Amin R, Ratjen F. Emerging drugs for cystic fibrosis. Expert Opin Emerg Drugs 2014; 19: 143-155.

6 MacKenzie T, Gifford AH, Sabadosa KA, et al. Longevity of patients with cystic fibrosis in 2000 to 2010 and beyond: survival analysis of the Cystic Fibrosis Foundation Patient Registry. Ann Intern Med 2014; 161: 233-241.

7 Kerem E, Viviani L, Zolin A, et al. Factors associated with FEV1 decline in cystic fibrosis: analysis of the ECFS Patient Registry. Eur Respir J 2014; 43: 125-133.

8 Stephenson AL, Tom M, Berthiaume Y, et al. A contemporary survival analysis of individuals with cystic fibrosis: a cohort study. Eur Respir J 2015; 45: 670-679.

9 Bell SC, De Boeck K, Amaral MD. New pharmacological approaches for cystic fibrosis: promises, progress, pitfalls. Pharmacol Ther 2015; 145C: 19-34.

10 Boyle MP, Bell SC, Konstan MW, et al. A CFTR corrector (lumacaftor) and a CFTR potentiator (ivacaftor) for treatment of patients with cystic fibrosis who have a phe508del CFTR mutation: a phase 2 randomised controlled trial. Lancet Respir Med 2014; 2: 527-538.

11 Simmonds NJ. Ageing in cystic fibrosis and long-term survival. Paediatr Respir Rev 2013; 14: Suppl. 1, 6-9.

12 Burgel PR, Bellis G, Olesen HV, et al. Future trends in cystic fibrosis demography in 34 European countries. Eur Respir J 2015; 46: 133-141. 